EVS27

Barcelona, Spain, November 17-20, 2013

\title{
Communication Solutions for a Cloud-Based Charging Management System for a Fleet of Shared-Use Electric Vehicles
}

\author{
Michael Mierau ${ }^{1}$, Simon Fey ${ }^{2}$, Robert Kohrs ${ }^{1}$, Christof Wittwer ${ }^{1}$ \\ ${ }^{1}$ Michael Mierau (corresponding author), Fraunhofer-Institute for Solar Energy Systems, Heidenhofstr. 2, 79110 \\ Freiburg, Germany, michael.mierau@ise.fraunhofer.de \\ 2Simon Fey, Offenburg University of Applied Sciences, Badstraße 24, 77652 Offenburg, Germany
}

\begin{abstract}
The paper proposes a system architecture for charging infrastructure that serves the requirements of future fleets of shared-use electric vehicles in urban scenarios. The focus of the development is on the interfaces to central stakeholders such as mobility service providers, distribution network operators and utilities. The main concept of the proposed system is the adherence to a stringent resource-oriented design approach, following the design principles of the Representational State Transfer (REST) architectural software style for distributed systems. This design approach is used from the cloud-based services down to the implementation of the charging infrastructure's control algorithms. Focusing on the resources of the various entities simplifies the implementation of their interactions, compared to the explicit declaration of services that are available. The system design ensures that the charging infrastructure is open to all users and generates a benefit beyond basic charging operations. Integration in emerging smart markets is done via open web-based interfaces. These allow for the generation of an added value of concrete services for shared-use electric mobility. A link to the field of grid operation is proposed using the ISO/IEC 61850 telecontrol standard. The smart meter capabilities of the charging stations can be used to gain additional information on the current state of the distribution grid. As an exemplary service a load management service for a fleet of shared-use electric vehicles is going to be implemented.
\end{abstract}

Keywords: communication, control system, fleet, load management, smart grid

\section{Introduction}

The widespread introduction of electric vehicles (EVs) is a major goal amongst policy makers because of their potential to significantly reduce the $\mathrm{CO}_{2}$-emissions of the transportation sector and thus the reliance on fossil fuels for transportation [1]. Especially interesting fields of application for EVs are urban areas, where benefits such as virtually no local exhaust and noise emissions by far outweigh their currently limited available range. Hence, many cities such as Amsterdam introduce strong incentive programs to support the introduction of EVs [2]. However, EVs are expensive due to high battery costs and limited in their usability compared to conventional vehicles. Because of that, private ownership is not going to generate a mass market in the foreseeable 
future. Instead fleet operators such as car2go or businesses electrifying their fleet for urban operation are to be considered the most interesting first movers [3]. The system presented in this paper therefore focuses on shared-use electric mobility, while still being open to be used by privately owned EVs.

The future fleet of EVs in urban areas is expected to be made up of very heterogeneous parts, ranging from privately owned EVs to large fleets of mobility service providers. The aggregated energy and power demand of those future fleets does have the potential to affect grid operation, especially when charging takes place simultaneously [4]. This can either happen chronologically, when many charging processes take place during peak load times, or spatially when certain areas of the distribution grid face an unusual high concentration of EVs. On a smaller scale future grid-connected micro-grids, which have to integrate numerous EVs into the local control algorithms, or parking areas with a high concentration of charging spots create the need for an intelligent grid integration of EVs. All these examples will be implemented by different stakeholders with the goal to resolve the local restrictions. Yet they need to be open to all vehicles in order to not exclude users from certain charging spots and thus endanger the acceptance of EVs.

The central component of any intelligent grid integration is the charging infrastructure. However, a solid business case for the implementation and operation of a widespread charging infrastructure is hardly available, since key requirements such as being accessible to all utilities and users alike tend to create costs that are not in relation to the achievable benefits. Each charging operation only generates a small transaction volume [5] and possible additional benefits, e.g. for grid operation, are not standardized and agreed upon today. Hence, the amortization of the investment costs of a widespread installation especially of public charging stations is going to be difficult [6]. Thus, an evolutionary development of the system is expected, with local charging solutions or clusters slowly growing into a city-wide charging infrastructure, which is capable of utilizing all of the potential benefits of electric mobility.

In this paper we introduce a charging infrastructure and its connection to a higher-level communication system that allows for the operation of a fleet of shared-use EVs. In section 2 we introduce the concept of the system and the interconnections between the various entities. Furthermore, we discuss potential benefits for the various involved stakeholders. In section 3 we provide an overview of the system design followed by the implementation and conclusion in section 4 and 5 , respectively.

\section{Concept}

The need to manage and even control charging processes that take place at various charging stations spread out over a large urban area requires the implementation of a large-scale communication infrastructure. Not only billing of charging processes requires communication but also approaches to coordinated control strategies, which eliminate the effects of simultaneous actions of various independent EVs. Our system builds on an architecture of open data platforms that operate in a cloud service environment, as presented in [7]. This data cloud is the key component for handling electric mobility data, the fundament that all emerging services and business models around shared-use electric mobility are based on. The charging infrastructure is connected to and interacts with the data cloud in order to open up the potential of EVs to the involved stakeholders. The potential benefit of the proposed system to these stakeholders is discussed below.

We consider six main stakeholders to be involved in a smart charging infrastructure for shared-use:

- EV manufacturer

Carmakers that entered the market for EVs, likely with models being specially suited to the needs of shared-use fleets. The integration of smart-charge functionalities into their models may give them an advantage over their competitors.

- Charging Infrastructure Operator (CIO)

The CIO maintains one or more charging stations (CS) in order to sell the service of charging. He can be seen as the technical operator and administrator of a network of charging stations, and takes care of the hardand software of the systems in the field. There may be various CIOs active in future urban areas.

- EV Fleet Operator (FO)

The Fleet Operator is responsible for a fleet of electric vehicles. His interest is to provide mobility to vehicle users.

- Distribution Network Operator (DNO) 
The DNO supplies electricity to the charging infrastructure. His responsibility is the electrical operation of the system and grid stability. Another interest of the DNO is the provision of value-added services by the demand side, such as peak load shifting or shedding inside his distribution network.

- Utility

Utilities may use the system to market and trade energy.

- Third Party Service-Provider

The cloud infrastructure opens up the possibility for third-party service providers to enter the market of shared-use electric mobility with new products based on the aggregated mobility data which is available in the cloud.

The potential benefit of our system for each stakeholder is regarded in the following subsections.

\subsection{EV manufacturer}

Modern vehicles do possess an ever rising number of interfaces and services for comfort and multimedia purposes, whereas intelligent charging solutions are not due to enter the market within the next years. However, new mobility concepts force the stakeholders of the automotive industry to expand the cooperation with fleet operators, grid operators and utilities [8]. The most advanced activity that stems from such cooperation is the standardization process of the vehicle to grid communication interface under the ISO technical committee 22 as drafted in the ISO/IEC DIS 15118, which defines the protocol and data representation for the communication between the charging infrastructure and the vehicle. The system proposed in this paper builds on this standard for a direct control of conductive charging processes. Apart from standardization activities there are currently mainly bilateral agreements between OEMs and utilities to work on the development of charging infrastructures [9]. So far these solutions focus on providing a reliable infrastructure and local energy management algorithms. The EVs themselves hereby only implement basic charging scheduling algorithms, since currently there exists no business case for an intelligent integration of EVs into the grid. However the inclusion of build-in intelligent control algorithms of future EVs into the system proposed in this paper is feasible and desired, depending on the use case to be realized.

\subsection{Charging Infrastructure Operator}

The charging infrastructure itself shall be open to any user and utility, since proprietary solutions in that field negatively affect the acceptance, evolution of services and widespread introduction of the system. As a result charging stations have to be as simple and easily configurable as possible while still providing a benefit beyond basic charging operations. The heterogeneous nature of the emerging market with various fleet operators, users and utilities all operating on a common charging infrastructure, which in turn consists of many sub-systems run by various CIOs, makes the implementation of any control algorithms in the charging stations themselves not feasible. The effort needed to run such a system is out of proportion to the achievable gains. Ideally the management routines are incorporated into either existing control entities, such as grid control centers, or into newly created cloud-based services that any stakeholder, e.g. a fleet operator, can easily commission to any third-party service provider. The charging station itself should only interact with one fixed backend unit. This backend unit shall provide the charging station with a charging schedule, which includes the reference input to be used to charge the connected EV. This central backend unit is run by the charging infrastructure operator himself, since this opens up the possibility to realize various use cases via an interaction of cloud services without having to reconfigure the charging stations all the time. This way a CIO is able to pool many charging stations and control their aggregated load in order to provide services like control energy. Furthermore he could forward requests for charging schedules to other management services, e.g. fleet energy management services implemented by fleet operators with big fleets.

\subsection{Fleet operator}

Fleet operators will benefit from a network of cloud-connected charging infrastructure not only to ensure that the mobility needs of their users are met. With an increasing size of their fleet and thus the aggregated energy demand, they can easily implement own charging control strategies that rely on the interconnectivity which is provided by the data cloud. These control strategies could then create charging schedules that depend on the availability of energy from renewable energy systems (RES). 


\subsection{Distribution Network Operator}

The distribution network operators (DNOs), who are responsible for safe and reliable grid operation, face the challenge to meet the demands of future fleets of EVs for charging power and energy. Although the German grid operators anticipate only very little need for an expansion in grid capacity for the inclusion of electric mobility prior to 2020, a transformation to smart grids can accelerate the introduction of market-based control mechanisms for EVs [10]. Such market-based control mechanisms are envisaged to handle all the issues surrounding the generation, conversion and sale of energy. Here, the grid itself is considered to take a serving role for the market and the grid operators are not allowed to actively take part in the ensuing market operations. In contrary, all the issues surrounding grid capacity and the provision of usable "line width" in the distribution grid are the core business of the DNO [10]. Given the significant load that future EVs with a charging power of up to $22 \mathrm{~kW}$ constitute, it is advisable to provide the DNO with a control interface to the charging infrastructure. That way he is enabled to assert direct control over charging processes in case of critical states in grid operation. This is especially important during early stages of the introduction of charging infrastructure, when temporary or regional supply shortages due to the additional load of EVs are not likely to be handled by smart market mechanisms.

Furthermore, any implementation of control strategies for the whole or parts of the distribution grid depends on reliable information on the current state of the grid. Charging stations can support the implementation of distribution grid supervision by providing measured data, which reduces the number of metering units to be installed in order to gather power grid state information.

\subsection{Further stakeholders}

An architecture of cloud-services based on open data as proposed in [7] would bring together additional stakeholders such as utilites, customers of mobility service providers and third-party service providers from various fields. This approach makes it easy for stakeholders like fleet operators to commission the operation of the necessary IT infrastructure or even the management algorithms to said third-party service providers, given that the charging infrastructure and the connected vehicles are able to interact with the system. As a result new business opportunities may arise from the introduction of such a system.

\section{System Design}

The charging infrastructure's main components are 1) an embedded computer system responsible for communication, application and local control operations and 2) a related periphery controller board for local hardware control. Both are described in section 3.1. Section 3.2 gives a detailed describtion of the implementation of the charging station's connection to the data cloud. Finally, section 3.3 outlines how a cloud-based energy or load management can be implemented for fleets of EVs.

Fig. 1 shows an overview of the proposed system and the communication interfaces between the various entities. Since the focus of this paper is on the charging infrastructure and its related higherlevel control entities, the IT-aspects of the cloud concept and its implementation are not covered in detail. The main concept of the proposed system is the adherence to a stringent resource-oriented design approach, following the design principles of the Representational State Transfer (REST) architectural software style for distributed systems. This design approach is used from the cloud-based services down to the implementation of the charging infrastructure's control algorithms. Every interaction between entities is organized as a request from a client that is processed and answered by a server. Focusing on the resources of the server simplifies the implementation of these interactions, compared to the explicit declaration of services that are available to a client.

The overview in Fig. 1 puts the charging infrastructure operator in the center, since his IT infrastructure plays a pivotal role for the handling of each charging process. Because of the clientserver-architecture of the system, the charging station needs a service that can be queried for reference input for a given charging process. This service is best assigned to the charging infrastructure operator. Based on the use case or business model to be realized, the CIO can create a suitable charging schedule, using optional further information from other stakeholders, or forward the whole request to a management service from another stakeholder. Approaches to the implementation of such control webservices are discussed in section 3.3. 


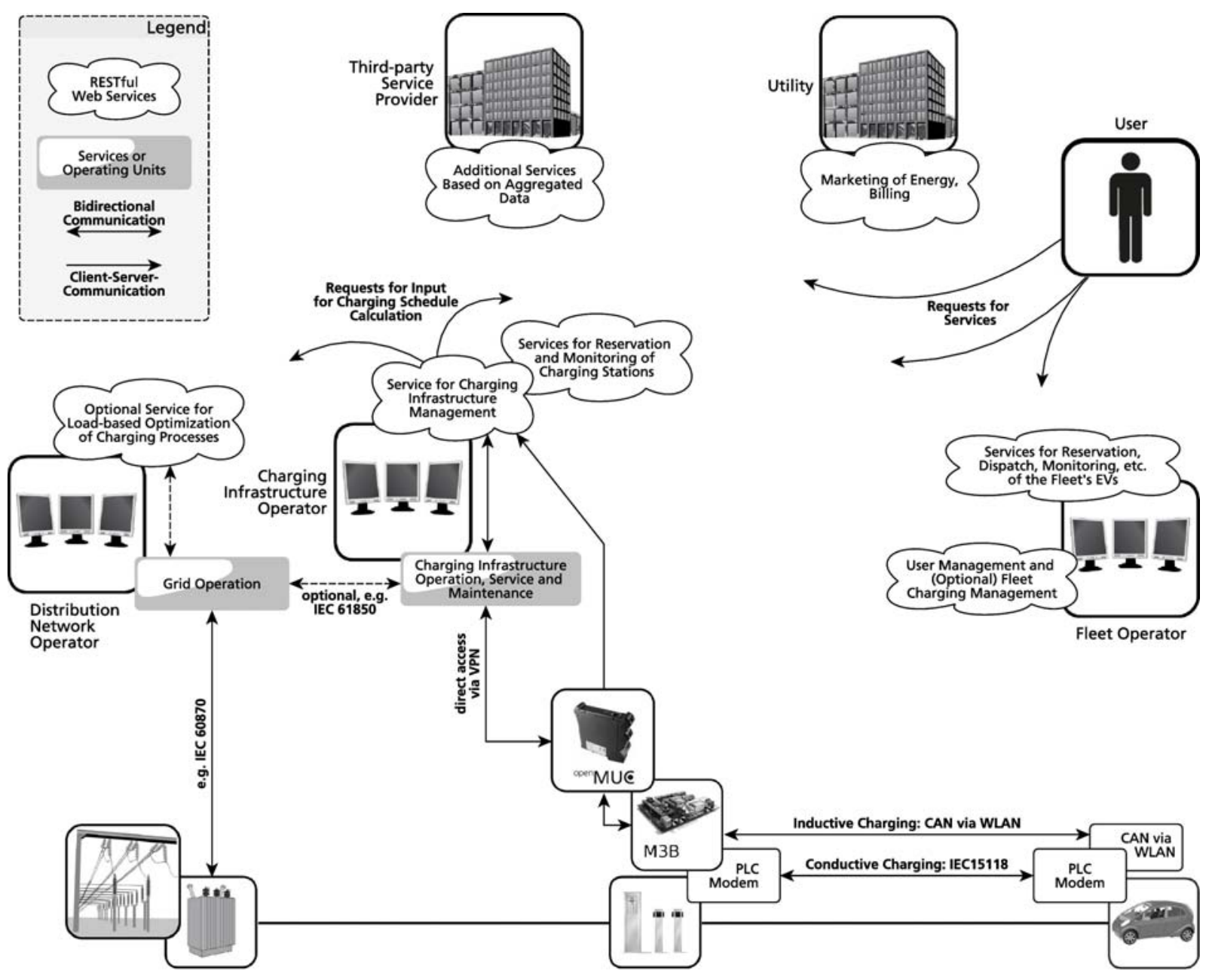

Figure 1: Overview of the system

The proposed system requires only the charging infrastructure operator to maintain direct access to the charging stations in the field. This direct access, which is expected to be required for service and maintenance anyway, can be used to implement further services on the CIO server. Possible services may be the implementation of telecontrol servers for the DNO, as described in section 3.2.4.

\subsection{Design of the charging stations and communication interfaces}

The charging station design follows a modular design approach both for the hard- and software components [11]. The use of basic system components that implement generalized tasks, such as metering or the handling of standardized interfaces and routines, significantly shortens the time needed to create a running system. This translates to a faster time to market. The resulting drawback of the charging stations not being highly integrated or optimized with regard to installation size or energy consumption is of minor relevance for their use as a research platform. The systems to be used in future real-world applications will improve on that and implement further findings from current research projects.

The key component of the charging station is an embedded Linux device with a lightweight modular software framework named OpenMUC ${ }^{1}$, which has been developed at Fraunhofer ISE for monitoring and control applications. The framework is run in JAVA/OSGi and requires the GNU classpath. It is designed to automatically handle the acquisition of data for a set of predefined metering or data channels. The lower part of Fig. 2 gives an overview of the internal structure

\footnotetext{
${ }^{1}$ http://www.openmuc.org/
} 
of the OpenMUC system. The Data Manager not only handles the acquisition of data from the field via the registered drivers, it also provides a service for user access control. In the proposed client-server-system this user access service is the gateway for incoming requests, either from the local application or from external entities. This way the whole periphery of the central device is made available to both, local flow control algorithms as well as remote higher-level control entities. The remote access is described in paragraph 3.2 in further detail.

The handling of conductive charging processes according to the relevant standard (IEC 61851-1 mode 3) requires the constant monitoring of security and status information and the provision of a pulse-widh modulated signal to the EV. This task is taken care of by a microcontroller-board called M3B, which has also been developed at Fraunhofer ISE [11]. It acts as a periphery controller to the OpenMUC-system, providing power-switching capabilities via on-board relais that switch external contactors and an additional Controller Area Network (CAN) interface. The latter is used to communicate directly with the vehicle during inductive charging, as described in paragraph 4.

\subsection{Connection of the charging stations to the data cloud}

The communications backend of the charging station, which enables the fleet optimization and system control, is realized with cloud technology. In the following we introduce the system architecture and components which realize the complete communication path, from the cloud server system down to the local fieldbus and hardware I/O.

Fig. 2 illustrates the OpenMUC system and local cloud-communication modules in the charging station. There are two implementations of REST web services: 1) the REST-client for backend communication with the server in the cloud and 2) an integrated REST-server for remote connections from the charging station operator.

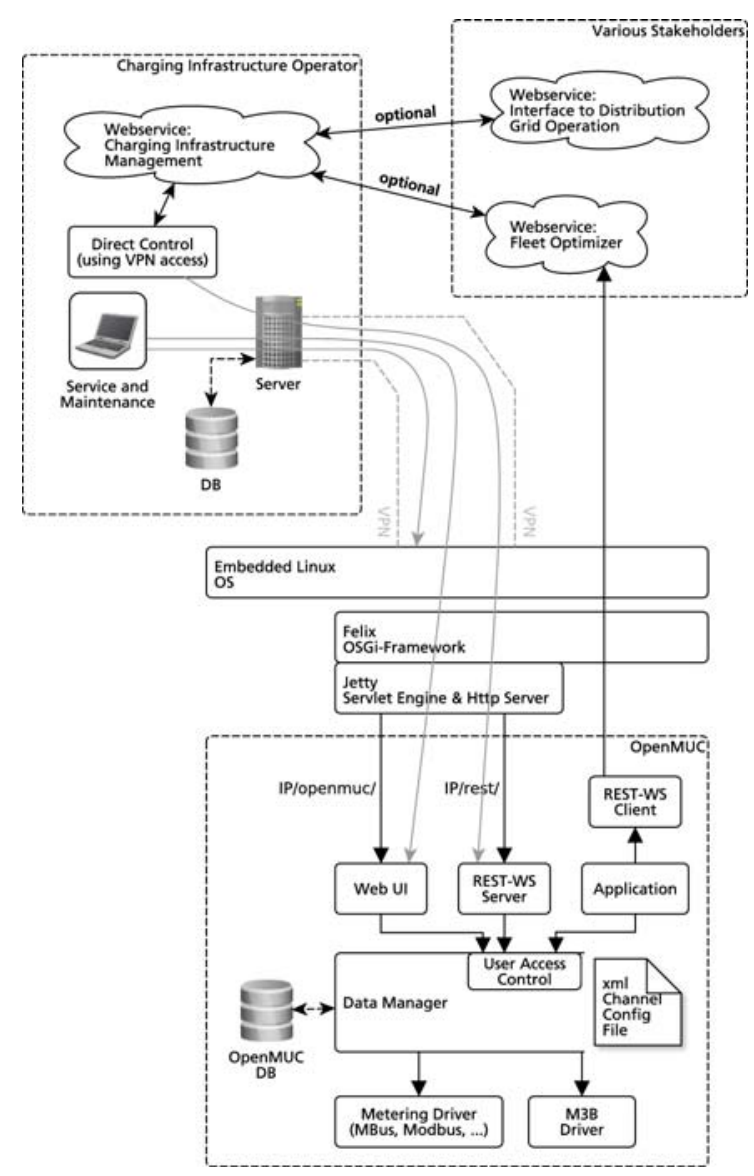

Figure 2: Overview of the OpenMUC system and higher-level entities

\subsubsection{REST-client to the cloud}

The client communication to the various cloud services is used to provide the main functionality of controlled charging. The charging station is client-only in this implementation. As described, the charging station requests the reference input at the CIO's server. In this initial request the charging station provides the CIO server with the user's credentials and identification and charging parameters provided by the EV. The CIO's service for charging infrastructure management can now interact with any related management services of this user. The CIO server answeres the request with a charging schedule that consists of a $\mathrm{P}(\mathrm{t})$ curve, which subsequently is communicated to the EV.

\subsubsection{REST-server for remote connections}

Secondly there is a REST-servlet registered in the local HTTP server for incoming connections from remote applications. This REST-server is only reachable from the secured Virtual Private Network (VPN) between the CIO server and his distributed charging stations. On this 
communication path the charging station operator/administrator can monitor and control the system behavior. According to the RESTbased system design, HTTP-PUT and HTTPGET operations are available, which both can be applied to the measurement data channels that are defined on the charging station. The channels are accessible using directory-like URIs. The incoming operations are coupled to synchronous blocking function calls that cause actions on the local attached components, e.g. when writing to a channel attached to the M3B periphery controller, and fieldbus devices.

\subsubsection{Data representation and control abstraction layer}

For representation of the exchanged data Java Script Object Notation (JSON) is used. Its design reduces the overhead in comparison to e.g. XML and still remains human-readable.

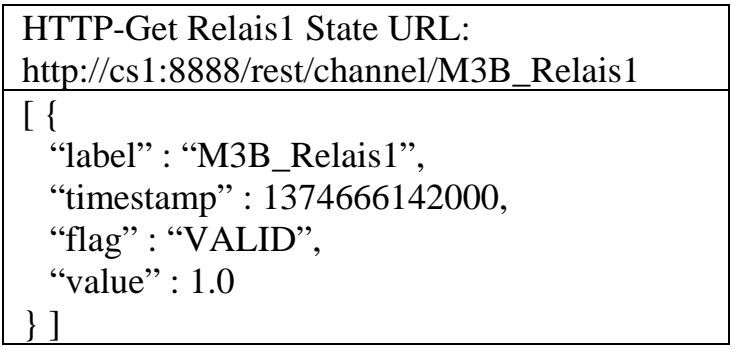

Table 1: HTTP-GET data representation for retrieving the state of the Relais1 on the attached M3B

Hardware I/Os, system actions and configurable parameters are represented as channels as shown in Table 1. Every channel has a unique identifier label that is mapped to the application in a configuration file. Channels support $\operatorname{read}()$ and write() operations and are exhibited to A) the REST-server which maps HTTP-GET and HTTP-PUT to the internal API and B) the internal API. This internal API, which is referred to as Data Manager, is the main interface used by the charging station application. Fig. 2 illustrates the OpenMUC control abstraction layers. Inside the Data Manager a protocol multiplexer called Driver Manager redirects read() and write() operations to the corresponding software module, which implements the protocol to access the hardware or fieldbus. This is transparent to the charging station application.

\subsubsection{IEC61850-Server for remote control access}

The security-critical task of safe grid operation may require a separate communication channel from the respective DNO's grid operation entity to the charging stations within his area of responsibility. For this communication the established telecontrol standard from the area of the transmission networks ISO/IEC 61850 with its draft IEC TR 61850-90-8, which defines object models for electric mobility, may be used. Although this draft is not yet regarded as being suitable for a market-based control of EVs [13], it may provide a straightforward way to integrate charging stations into grid operation. Currently the ISO/IEC 61850 standard is used for monitoring and control of RES like wind turbines, using the data models specified in the IEC 61400-25. These existing monitoring systems could be built upon to also integrate EV charging processes. Given the system structure described with charging stations being clients only, the interface for the DNO would be the CIO server. Here, an IEC 61850 server could be implemented which maps incoming requests to REST-requests to the charging stations in the field.

This system offers the potential for the distribution grid operator not just to assert control over charging processes in case of an emergency in grid operation, but also to gather further information on the operational state of the grid. This additional information can potentially boost the possible functionality of control algorithms which are implemented on the DNO side.

\subsection{Cloud-based load and/or energy management}

As described in 3.2.1, the charging stations are clients that request a reference input from a cloud service in order to control the charging process. Fig. 3 shows an overview of possible cloud services for the scenario of urban shared-use electric mobility. The horizontal classification divides the services into the layers of data services, services for reservation and availability and valueadded services. The vertical classification proposes assignments of services to stakeholders, which are most likely to implement and offer the respective service. This simplified overview is meant to show possible interactions between various services from different stakeholders in order to implement an intelligent grid integration of future fleets of EVs. Cloud-internal mechanisms such as authorization and data access management are not depicted. Data services and reservation and availability services can be seen as portals providing access to the data in the data cloud. Their use depends on the business case or use case 


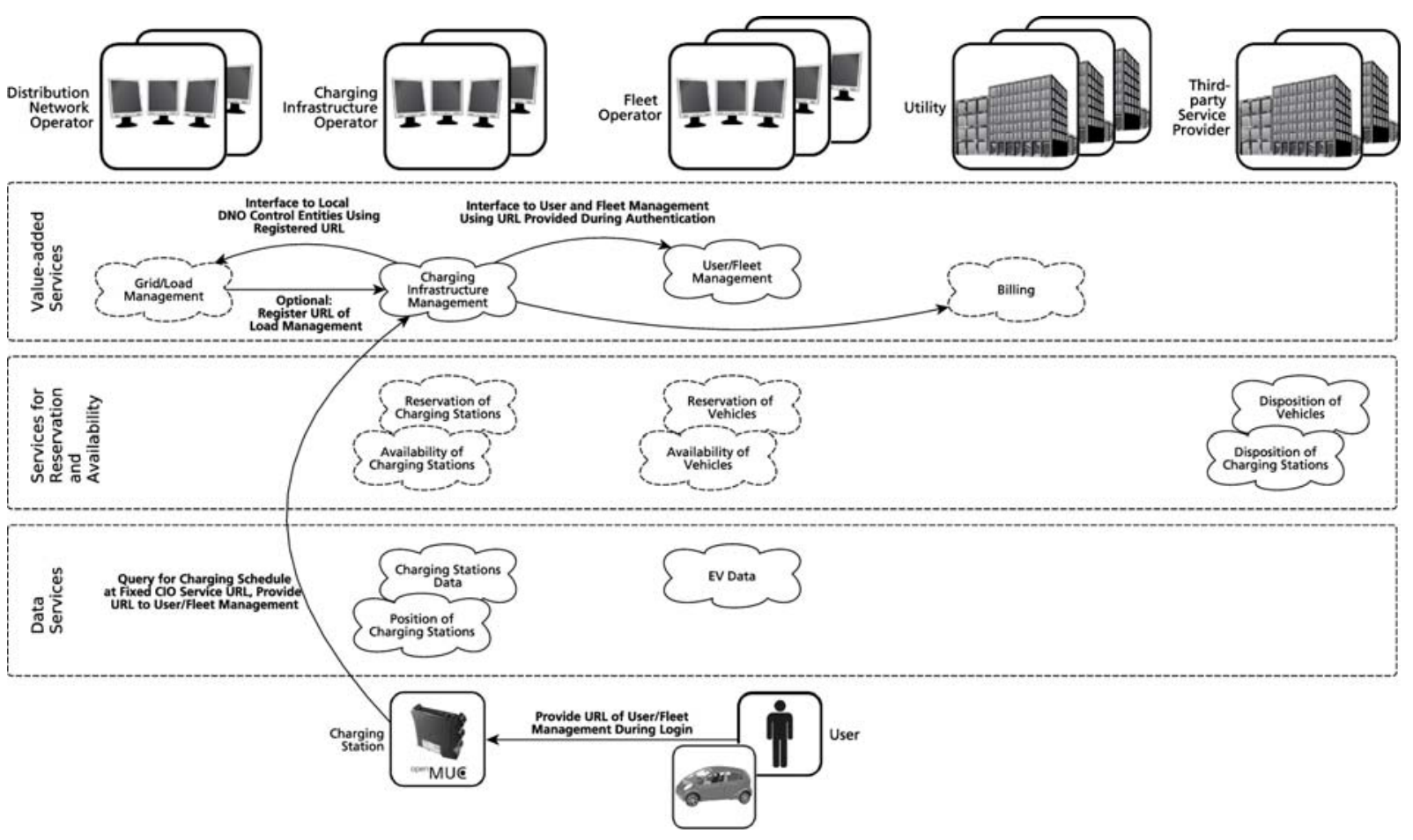

Figure 3: Cloud-based charging management

to be realized. Although mainly provided for services for the user they can also be included in the charging management.

The local control algorithm in the charging station accepts the user's identification details and requests a charging schedule for this user at the CIO server. If any dedicated control service is to be used for this specific customer, then the charging station is presented with an URL, which in turn is provided to the CIO charging infrastructure management along with the request for a charging schedule. This schedule consists of tuple of timestamps and reference power values. It is the result of an interaction of various cloud services, depending on the involved stakeholders and the details of the implementation. As described in section 2.1, current EVs rarely implement an intelligent charging strategy based on the reference values they receive at a charging station. The aforementioned ISO/IEC DIS 15118 defines a use case, in which the EV is given a curve of $P_{\max }(t)$-values and a tariff curve. Based on this information a charging schedule is created by the EV and subsequently communicated to the charging station. If accepted, the contactor in the CS is closed and the EV starts the charging process. Given the basic charging scheduling functionality of current EVs, a modified $\mathrm{P}_{\max }(\mathrm{t})$-curve is used to trigger the creation of a schedule that is consistent to the requirements of the stakeholders.

This way the CIO server acts as the primary communication link to the control services of the various stakeholders. The implementation of specific control routines by any given stakeholder requires a concept for a business case in order to return the necessary investments. Electric mobility constitutes an emerging market with business models that are still in the making. This is one reason for creating a flexible environment such as the proposed cloud-connected charging infrastructure as the fundament for the evolution of future business models. In the following possible control approaches for two stakeholders are considered: fleet operators and distribution grid operators.

Intelligent charging solutions providing demand side management or even vehicle to grid mechanisms could be implemented by fleet operators with a large fleet and thus a large energy demand to be met. Especially the supply of charging EVs with energy from fluctuating RES is a task that justifies the implementation of a cloud service for charging management. Depending on the business case to be realised, the reference input can be a financial incentive or a tariff signal provided by the fleet operator's utility. This tariff 
would signal the current availability of energy from RES. Such a control of charging processes with regard to the energy demand of the EVs constitutes a smart market mechanism.

The distribution grid operator has to ensure safe and reliable grid operation. Depending on the characteristics of a given urban distribution grid and the number of EVs using it, the need to implement load management routines may arise in order to avoid the costly expansion of the grid. EVs lend themselves to an integration in such grid capacity control strategies, because their power demand can easily be adjusted or managed according to the current state of the grid. As described in section 2, the management of grid capacity is not suitable to be handled with smart market mechanisms. The natural monopoly of the grid operator hampers the introduction of solutions based on business competition. On the other hand, the provision of input for smart market mechanisms, that signals the current state of the grid, such as load-variable grid charges, is considered to be too complex to be efficiently utilized for grid capacity management [10]. However, the introduction of reduced grid charges for loads that can be controlled within a central capacity management, such as EVs and heat pumps, may provide the possibility of refunding grid-friendly charging behaviour.

Access to the charging infrastructure can be implemented in various ways. Section 3.2.4 describes an approach that is shown in Fig. 1, where the CIO provides a communication server that implements an established telecontrol protocol, e.g. IEC 61850. The DNO can now use this server to provide the charging schedule creation entity of the CIO with input for the grid situation or even concrete reference input. Fig. 3 shows an alternate approach using a dedicated cloud service, implemented by the DNO. With the knowledge of the charging stations' position in the grid, the DNO may register this service at the respective CIOs' management services, so that requests from certain charging stations are answered with a schedule that has been created together with the DNO.

In both scenarios, a cloud-based optimization algorithm would take the input variables from a list of pending and ongoing charging processes, such as energy demand, maximum charging power and planned time of departure, and try to meet the EV's energy demand within predefined limitations. The limitations result from the maximum possible charging power of the various charging stations and from the use case to be implemented. A fleet operator might try to control the aggregated energy demand of his fleet so that it matches the prognosed generation from RES such as PV and wind. The DNO on the other hand would try to minimize the aggregated charging power or try to shift charging processes out of times with peak demand. The data cloud would always act as the main enabler for such control approaches, since it provides the interconnectivity needed in order to communicate the reference input to the stations in the field.

\section{Implementation}

The main components of the proposed system the charging infrastructure and cloud-based charging management - are implemented in the context of the research project "Shared Use of EMobility: Vehicles, Data and Infrastructure"2. The project focuses on the energetic and IT interfaces between users, vehicles and infrastructures of future urban mobility concepts revolving around shared-usage of mobility resources. A central part of the project is the implementation of a demonstrator system, in order to gain hands-on experiences with the various technological tasks, such as disposition and navigation cloud services, vehicle positioning systems and an inductive charging infrastructure. The latter is developed in order to address the topic of a seamless integration of charging infrastructure into urban areas. The charging station demonstrator features an OpenMUC-based application that directly controls the $22 \mathrm{~kW}$ inductive charging system and that is connected to the higher-level control structures as presented in this paper. The first prototype of the demonstrator system is due in December 2013.

\section{Conclusion}

The paper proposes a reference design for a charging infrastructure for future urban mobility concepts based on communication interfaces and information technology that allows various stakeholders to participate in the emerging market of shared-use electric mobility. The main enabler for this market to develop is the data that is acquired, shared and processed via the various services of the mobility data cloud. Improving the access to such data for various stakeholders enables the development of services that provide mobility, the disposition of resources such as EVs

\footnotetext{
${ }^{2}$ http://www.gemo.fraunhofer.de/
} 
and charging stations and higher-level control algorithms for an optimized operation of the system.

The consequent adherence to a resource-oriented system design according to the REST design principles results in a system with charging stations being both, clients to cloud-based webservices on the one hand and servers for direct operational access on the other hand. This system is way more flexible to the field of application. Direct charging station control requires a bidirectional communication system for a fast and responsive system. Centralized client-server cloud-architectures are limited due to the request-response communication manner. The bidirectional cloud communication with REST-client and REST-server offers control interfaces to parties with different interests from car sharing, fleet-management and future powergrid services such as voltage control and virtual power plants.

\section{Acknowledgments}

The research presented in this paper is carried out within the scope of the research project "Shared Use of E-Mobility: Vehicles, Data and Infrastructure", which is a joint project by six Fraunhofer Institutes financed by the "Markets Beyond Tomorrow"-program of the FraunhoferGesellschaft.

\section{References}

[1] BRD, Die Bundesregierung: German Federal Government's National Electromobility Development, Die Bundesregierung, 2009

[2] Amsterdam electric, http://www. amsterdam.nl /parkeren-verkeer/amsterdamelektrisch/amsterdam-electric, accessed on 2013-02-14

[3] Car2Go Amsterdam, http://www. car2go.com/en/amsterdam/\#, accessed on 2013-02-14

[4] T. Döscher, B. Wille-Haussmann and J. Link, Evaluation of Technical Integration of Electric Mobility into the Grid, presented at the EVS24, Stavanger, Norway, 2009

[5] C. Bleijs, Low-cost Charging Systems With Full Communication Capability, presented at the EVS24, Stavanger, Norway, 2009

[6] F. Kley, D. Dallinger and M. Wietschel, Assessment of Future EV Charging
Infrastructure, presented at the IAMF 2010, Geneva, Switzerland, 2010

[7] Tcholtchev, N.; Farid, L.; Marienfeld, F.; Schieferdecker, I.; Dittwald, B.; Lapi, E., On the interplay of open data, cloud services and network providers towards electric mobility in smart cities, Local Computer Networks Workshops (LCN Workshops), 2012 IEEE 37th Conference on , vol., no., pp.860,867, 22-25 Oct. 2012

[8] F. Kuhnert et Al., Zukunft in Bewegung. Die Automobilindustrie im Spannungsfeld zwischen neuen Chancen und alten Strukturen, Stuttgart/Hannover, PricewaterhouseCoopers, 2009

[9] R. Petry and A. Kainer, Elektromobilität Wachstumsinpuls für Energieversorger, Wien, RolandBerger, 2009

[10] BRD, Die Bundesnetzagentur: "Smart Grid" und "Smart Market", Eckpunktepapier der Bundesnetzagentur zu den Aspekten des sich verändernden Energieversorgungssystems, Die Bundesnetzagentur, Bonn, 2011

[11] M. Wieser, J. Clement, P. Benoit, R. Kohrs, C. Wittwer, A Modular Prototyping Hardand Software Platform for Faster Development of Intelligent Charging Concepts for Electric Vehicles, Power Engineering, Energy and Electrical Drives (POWERENG), $20134^{\text {th }}$ IEEE IES International Conference on, 13-17 May, 2013

[12] E. Lapi, N. Tcholtchev, L. Bassbouss, F. Marienfeld, I. Schieferdecker, Identification and Utilization of Components for a Linked Open Data Platform, Computer Software and Applications Conference Workshops (COMPSACW), 2012 IEEE 36th Annual, vol., no., pp.112,115, 16-20 July 2012

[13] E. Nauck, E. Oswald, M. Spähn, Application of standard IEC 61850 in smart grids and their benefit in the field of electromobility, presented at the VDE Kongress "Smart Grid" 2012, Stuttgart, Germany, 2012

\section{Authors}

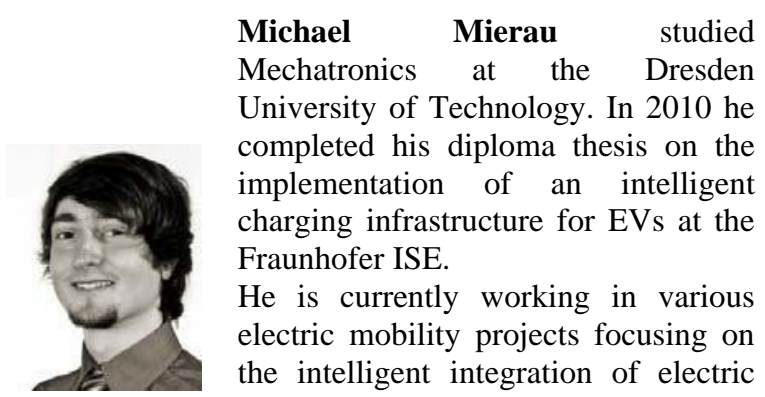


vehicles into the grid.

Simon Fey finished his Master Degree in Electrical Engineering / Information Technology at Offenburg University of Applied Science in 2010. He wrote his thesis at Fraunhofer ISE on acquisition and storage of measurement data from power plants. Simon Fey is a member of graduate school "KleE - Small scale renewable energy systems" and currently writing his $\mathrm{PhD}$ thesis under the supervision of Prof. Dr. Andreas H. Christ. His thesis is on IT-based communication structures and system architectures for renewable energy systems.

Dr. Robert Kohrs studied physics in Bonn, Germany, and obtained his PhD in semiconductor detector physics in 2008. Since 2009 he is project manager in the smart grids research group of Fraunhofer ISE.

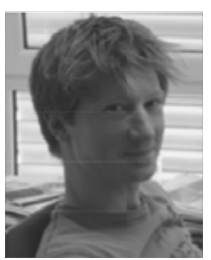

In 2012 he became head of the Group Smart Grid Technology. His main fields of research are communication technologies and protocols in power systems with a high share of distributed and renewable generation.

Dr.-Ing. Christof Wittwer studied electrical engineering at the University Kaiserslautern. In 1999 he received his $\mathrm{PhD}$ at the University of Karlsruhe. His research activities focused on the development of a dynamic system simulator which is used for the control

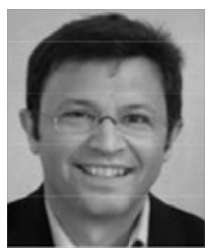
deployment of thermal energy systems.

Since 2003 he is leading the Group "Control Devices BSR" at Fraunhofer ISE, since 2010 he is head of the Department "Smart Grids". He is lecturer at Freiburg University. 